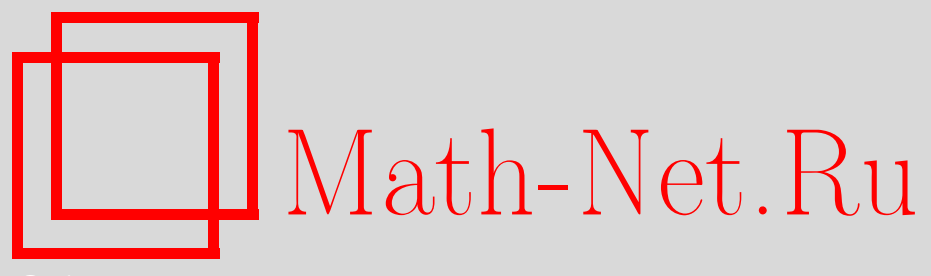

В. П. Маслов, Общее понятие топологических пространств отрицательной размерности и квантование их плотностей, Матем. заметки, 2007, том 81, выпуск 1, 157-160

DOI: https://doi.org/10.4213/mzm3530

Использование Общероссийского математического портала Math-Net.Ru подразумевает, что вы прочитали и согласны с пользовательским соглашением http://www.mathnet.ru/rus/agreement

Параметры загрузки:

IP : 54.210 .77 .194

26 апреля 2023 г., 14:45:58

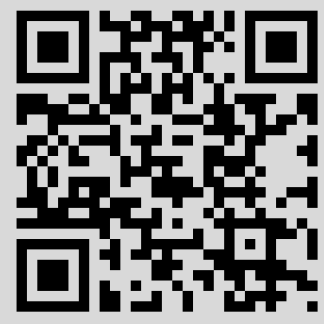




\section{Общее понятие топологических пространств отрицательной размерности и квантование их плотностей}

\section{В. П. Маслов}

В данной статье, которая является в некотором смысле дополнением и продолжением статьи [1], мы введем общее понятие топологических пространств отрицательной размерности (в отличие от отрицательной размерности асимптотических пространств в [1]).

Общее определение пространств отрицательной размерности. Пусть имеется компакт $M_{t_{0}}$ хаусдорфовой размерности $t_{0}$, являющийся элементом $t$-параметрической шкалы вложенных друг в друга компактов $0<t<\infty$. Эти шкалы будем называть эквивалентными относительно $M_{t_{0}}$, если у них совпадают компакты при $t \geqslant t_{0}$. Будем говорить, что компакт $M_{t_{0}}$ является дыркой в этом эквивалентом множестве шкал, $\mathrm{a}-t_{0}$ есть отрицательная размерность этого класса эквивалентности.

Примеры. Червяк проедает в стволе дерева сложную извилистую дорожку-дырку. Она настолько меньше ствола, что мы можем считать, что толщина ствола бесконечно велика по сравнению с дыркой.

Размерность проволочки той же толщины и точно такой же "извилистости" мы можем определить независимо от окружающей среды, а дырку, проделанную червячком, нельзя рассматривать вне ствола, хотя обе размерности совпадают по абсолютной величине.

В теории кристалла Френкеля дырка - это отсутствующий электрон, и она ведет себя как частица, двигающаяся вдоль кристалла, но в отличие от электрона не может существовать вне кристалла - в пустоте дырки нет. Точно так же дырка в нашем понимании не может существовать вне достаточно большой шкалы, в которой она "проделана".

Реально фрактальная размерность дырки, трещины в горной породе и т.п. зависит от масштаба, в котором "наш глаз" различает точки. Так, береговая линия будет иметь фрактальный характер, когда мы ее наблюдаем с самолета. Если же мы находимся непосредственно на берегу, то эта линия будет гладкой и ее размерность будет равна размерности гладкой линии, т.е. единице.

Если мы рассматриваем трещины в металле, то размерность зависит от разрешающей способности прибора, через который мы наблюдаем трещину [2]-[4]. Обозначим линейную разрешающую способность прибора через $\kappa$. Обычное определение размерности Хаусдорфа основывается на стремлении к нулю некоторых шаров, на практике, как правило, трехмерных. Это означает, что объем $\Omega$ рассматриваемого металла должен быть много больше, чем $\kappa^{3}$. Таким образом, приведя задачу к единичному объему, мы получим, что радиус хаусдорфова шара (т.е. шара, который используется в определении размерности Хаусдорфа; см. [5]) $\delta=\kappa^{3} / \Omega$ стремится к нулю при $\Omega \rightarrow \infty$.

Далее возникает вопрос, как в конкретных задачах на щели понимать шкалу компактов, размерность которых $t$ увеличивается при $t \rightarrow \infty$. Размер-объем металла или горной породы не является размерностью: они трехмерные. Где возникает достаточно большая величина размерности?

Рассмотрим для сравнения газ в трехмерном сосуде. Каждая молекула газа движется. Число молекул $\approx 10^{23}$. И если рассматривается движение каждой молекулы, то, как правило, считают размерность равной $10^{69}$.

С другой стороны, можно рассматривать все молекулы как точки трехмерного пространства. И если еще учесть их перемещение за время $t$, то мы можем их рассматривать

Работа выполнена при поддержке Российского фонда фундаментальных исследований, грант № 05-01-00824. 
в четырехмерном пространстве. Именно эта двойственность позволила нам (с моим соавтором П. П. Мосоловым) перейти от уравнений Ньютона каждой молекулы к уравнениям полевидной гидродинамики всего газа [6], [7].

Если мы зафиксировали два момента времени расположения молекул газа в $10^{69}$-мерном пространстве, т.е. рассматриваем расположение всех $10^{23}$ молекул как одну точку в первый момент времени и как другую точку во второй момент времени, то можем определить естественное расстояние между этими точками, т.е. метрику.

Между этой метрикой и метрикой в трехмерном пространстве можно построить шкалу метрик, объединяя различные группы частиц.

Металлы, как и горные породы, с точки зрения механики (теории упругости) и тех масштабов, которые характерны для этой науки, состоят из “зерен” достаточно малого (но не атомного) размера-объема. Эти зерна могут быть разной величины, и мы можем их множество считать точкой в пространстве достаточно большой размерности [8], [9]. Именно такая трактовка позволяет нам перейти к общему определению фрактальной щели как дырки и увязать абстрактное математическое определение с реальными механическими объектами.

Распространение дырка-волна особенно наглядно видно при рассмотрении волн упругости в средах, в которых модуль Юнга на сжатие достаточно велик, а на растяжение равен нулю. В частности, таким свойством обладает песок. Волны-дырки подробно изучены автором совместно с П. П. Мосоловым в случае одной пространственной координаты [10; приложение].

Приведем теперь экономический пример.

Допустим человек получил огромное наследство в различных формах и растрачивает его бездумно "направо и налево". Если объем наследства вычислить сложно, то объем трат, возрастающих со временем $t$ как $t^{k}$ (аппетит трат может возрастать, как и аппетит наживы!), то $k$ - это отрицательная размерность, или $k$ - это размерность образующейся "дырки".

Рассмотрим теперь простейшие примеры мер (Хаара) в общем случае для $n$-мерных пространств. Пусть $S_{n}$ есть $n$-мерный шар радиуса $r$. В сферических координатах его объем

$$
\mu\left(S_{n}\right)=\operatorname{const} \int_{0}^{1} r^{n-1} d r=\operatorname{const} r^{n}
$$

здесь $r^{n-1}-$ плотность.

Умножение на координату двойственно в смысле преобразования Фурье дифференцируемости. Поэтому мы можем говорить о двойственных $n$ раз дифференцируемых функциях соболевского пространства $W_{2}^{n}$. "Сопряженным" к такому пространству является пространство $W_{2}^{-n}$ обобщенных функций по Соболеву.

Точно так же мы можем определить через "скалярное произведение" функции $W_{2}^{s}$, где $s$ - нецелое положительное, и $W_{2}^{-s}$ как "скалярно" сопряженное к нему.

Аналогично можно поступить с плотностью или весом $r^{s}$ и $r^{-s}$, используя, например, для представления функций из $W_{2}^{s}$ ядро Рисса или бесселев потенциал.

Недавно Ю. И. Манин в более общей форме и даже для комплексных $s$ применил эти соображения для плотности пространств с отрицательной размерностью [11].

Приведем пример пространств нецелой положительной размерности с мерой Хаара $r^{\sigma}$, где $0 \leqslant \sigma \leqslant 1$.

На отрезке $0 \leqslant x \leqslant 1$ существует шкала $0 \leqslant \sigma \leqslant 1$ канторовой пыли с мерой Хаара, равной $x^{\sigma}$ отрезка $(0, x)$, подобного всему данному множеству канторовой пыли. Прямое произведение этой шкалы на евклидов куб размерности $k-1$ даст всю шкалу $k+\sigma$, где $k \in \mathbb{Z}, \sigma \in(0,1)$. Именно относительно этой шкалы мы рассматриваем пространство отрицательной размерности $-D=-k-\sigma$. "Квантование" плотностей, рассмотренных в [11], приводит к плотности $C_{l}^{D+l}$. 
Как мы видели в [1], для понятия дискретной плотности отрицательной “дискретной” размерности надо рассмотреть определение типа распределения Бозе-Эйнштейна.

Как это рассматривалось в [1], относительно значений случайной величины $\lambda_{1}, \ldots, \lambda_{s}$ устанавливается упорядоченность по их величине. Среди чисел $\lambda_{1}, \ldots, \lambda_{s}$ могут быть и равные. В последнем случае их объединяют, складывая при этом отвечающие им "вероятности", т.е. отношение числа "попаданий" на $\lambda_{i}$ к общему числу испытаний. Число равных $\lambda_{i}: \lambda_{i}=\lambda_{i+1}=\cdots=\lambda_{i+k}$ будем называть кратностью $q_{i}$ значения $\lambda_{i}$. В нашем рассмотрении к бесконечности стремится как число испытаний $N$, так и $s$.

Пусть $N_{i}$ - число "выпадений" значения $\lambda_{i}: \lambda_{i}<\lambda_{i+1}$; тогда

$$
\sum_{i=1}^{s} \frac{N_{i}}{N} \lambda_{i}=M
$$

где $M$ - математическое ожидание.

Кумулятивная вероятность $P_{k}$ есть сумма первых $k$ вероятностей в последовательности $\lambda_{i}$ :

$$
P_{k}=\frac{1}{N} \sum_{i=1}^{k} N_{i}
$$

где $k<s$. Обозначим $N P_{k}=B_{k}$.

Если все варианты, для которых

$$
\sum_{i=1}^{s} N_{i}=N
$$

и

$$
\sum_{i=1}^{s} N_{i} \lambda_{i} \leqslant E, \quad E=M N \leqslant N \bar{\lambda}
$$

где

$$
\bar{\lambda}=\frac{\sum_{i=1}^{s} q_{i} \lambda_{i}}{Q}, \quad Q=\sum_{i=1}^{s} q_{i},
$$

$C>0$ - константа, равноценны (равновероятны), то [12]-[14] большинство вариантов будет скапливаться около следующей зависимости "кумулятивной вероятности"

$$
\begin{gathered}
B_{l}\left\{N_{i}\right\}_{i=1}^{l}=\sum_{i=1}^{l} N_{i}, \\
\sum_{i=1}^{l} N_{i}=\sum_{i=1}^{l} \frac{q_{i}}{e^{\beta^{\prime} \lambda_{i}-\nu^{\prime}}-1},
\end{gathered}
$$

где $\beta^{\prime}$ и $\nu^{\prime}$ определяются из условий

$$
B_{s}=N, \quad \sum_{i=1}^{s} \frac{q_{i} \lambda_{i}}{e^{\beta^{\prime} \lambda_{i}-\nu^{\prime}}-1}=E,
$$

если $N \rightarrow \infty$ и $s \rightarrow \infty$.

Для того, чтобы определить кратности $q_{i}$ в формуле (4), мы подобно тому, как поступали в [1], рассмотрим $D$-мерный квантовый осциллятор с частотой $\omega$. Его спектр, как известно [15], имеет вид

$$
\lambda_{i}=\left(i+\frac{1}{2}\right) h \omega
$$

где $h$ - постоянная Планка. Кратности [1] равны

$$
C_{i}^{D+i}=\frac{(G+i) !}{i ! D !} .
$$


Если переходить к единичному масштабу в формуле (4), т.е. положить $i / s=x$, то $0 \leqslant$ $x \leqslant 1$ и сумма при $s \rightarrow \infty$ будет стремиться к интегралу Римана.

Тем самым, плотности (6) являются квантованием плотностей в интегральных формулах Ю. И. Манина [11]. В целых точках $i=1, \ldots, D$ у (6) есть полюсы, имеющие смысл конденсата. Так, для распределения кратеров на Луне по их радиусу имеется четыре полюса (конденсат из четырех значений), что означает, что размерность, отвечающая их распределению, равна -4 .

Кратности $q_{i}$ являются аналогом плотностей в интегральной формуле и, следовательно, связаны с размерностью. В пределе при переходе от сумм к интегралам в формуле (4) мы получим, как это было показано в [1], плотности $r^{n-1}$, о которых говорилось выше. В этом смысле важно понять, как возникают эти плотности из рядов, т.е. из дискретного аналога. Этим самым мы как бы квантуем эти интегральные плотности Ю. И. Манина [11].

\section{СПИСОК ЦИТИРОВАННОЙ ЛИТЕРАТУРЫ}

[1] В.П. Маслов, Матем. заметки, 80:6 (2006), 856-863. [2] V.E. Saouma, C. C. Barton, J. Engrg. Mech., 120:4 (1994), 835-854. [3] Р. В. Гольдштейн, А. Б. Мосолов, ПММ, 56:4 (1992), 563-571. [4] Р. В. Гольдштейн, А. Б. Мосолов, Докл. РАН, 329:4 (1993), 429-431. [5] Б. Мандельброт, Фрактальная геометрия природы, Институт компьютерных исследований, М., 2002. [6] В. П. Маслов, П. П. Мосолов, Изв. АН СССР. Сер. матем., 42:5 (1978), 1063-1100. [7] В. П. Маслов, Квантование термодинамики и ультравторичное квантование, Институт компьютерных исследований, М., 2001. [8] R. H. Dauskardt, F. Haubensak, R. O. Ritchie, Acta Metallurgica et Materialia, 38:2 (1990), 143-159. [9] V.E. Saouma, C. C. Barton, N. A. Gamabeldin, Engrg. Fracture Mech., 35:1-3 (1990), 47-53. [10] V.P. Maslov, P. P. Mosolov, Nonlinear Wave Equations Perturbed by Viscous Terms, Walter de Gruyter, Berlin-New York, 2000. [11] Yu. I. Manin, "The notion of dimension in geometry and algebra", Bull. Am. Math. Soc., New Ser., 43:2, 139-161; arXiv: math.AG/0502016v1. [12] В.П. Маслов, Матем. заметки, 78:3 (2005), 377-395. [13] В. П. Маслов, Матем. заметки, 78:6 (2005), 870-877. [14] В. П. Маслов, Матем. заметки, 80:2 (2006), 220-230. [15] Л. Д. Ландау, Е. М. Лившиц, Квантовая механика, Наука, М., 1976.

\section{В. П. Маслов}

Поступило

Московский государственный университет

06.12.2006

им. М. В. Ломоносова

E-mail: v.p.maslov@mail.ru 